\title{
CONTINUOUS AND DISCRETE SLIDING MODE CONTROL OF AN ACTIVE CAR SUSPENSION SYSTEM
}

\author{
Jan Łuczko, Urszula Ferdek \\ Cracow University of Technology, Faculty of Mechanical Engineering, Kraków, Poland \\ e-mail: jluczko@mech.pk.edu.pl; uferdek@mech.pk.edu.pl
}

\begin{abstract}
In this paper, sliding mode control (SMC) algorithms are tested for their use in an active car suspension system. Using the quarter model of the car as an example, the comparison of the efficiency of the algorithms is made. A continuous and two discrete versions of the sliding mode control are taken into consideration. The study is limited to finding the relation between the control parameters and the comfort factor. This is done by analyzing the response of the model to the harmonic and impulse excitation.
\end{abstract}

Keywords: sliding mode control, active damping, car suspension

\section{Introduction}

The most common types of suspension systems used in automobiles are passives ones. Their construction is based on several spring and damping elements with constant, linear or nonlinear characteristics. When designing a passive car suspension, it is necessary to choose the optimal damping coefficient that would allow both the comfort and safety to be on a high enough level (Euczko and Ferdek, 2012). An alternative option is to use suspension which can modify its properties during ride of the vehicle - namely an active or a semi-active system. In the case of active suspension, the spring and damping elements are replaced with servomotors. This way the force generated in the suspension can be used to influence both the comfort and safety of the ride. Although active suspension systems are more complex and require more energy, they are more and more often considered and included in designs of modern vehicles.

One of the possible options for the active suspension control is to use a sliding mode control (SMC) algorithm (Yoshimura et al., 2001; Sam et al., 2004; Lin et al., 2009). The idea of the algorithm is to guide the model in the state-space, to a specific plane called "the sliding plane" and to keep it as close to this plane as possible. Therefore, two separate phases of this control can be listed: the "approach" phase, which lasts until the point describing the dynamics of the system reaches the switching hyperplane, and the "sliding" phase. In the second phase, the system is forced to "slide" along a slinging plane up to the desired point by using a discontinuous control signal. Most often, it is assumed that the sliding plane equation is linear and related to the regulation error.

The application of the sliding mode control to a physical system is accompanied by the so-called "chattering" effect (Lee and Utkin, 2007) - high-frequency oscillations which might cause wear or damage to the actuator. It is therefore essential to eliminate this effect from the regulation process.

As most of modern control systems are implemented as digital, below a procedure for designing a discrete sliding mode controller (DSMC) for control of a car suspension is presented. The most important advantages of the digital control are reduction of device cost, possibility to implement complicated control rules and higher control precision. 
In this study, a quarter model of the car is used for the purpose of testing the efficiency of damping the vibration by application of different control strategies. The performance factor includes parameters related to comfort of the ride.

\section{Model of the system}

The analysis has been performed on a quarter model of the car (Huang and Chen, 2006; Rajeswari and Lakshmi, 2008; Snamina et al., 2011) consisting of a non-spring-supported mass $m_{w}$ (mass of the wheel, axle and some elements of the drive transmission) and a spring-supported mass $m_{b}$ (1/4 of the other mass, mostly the car body). It has been assumed that the road influence on the wheel can be descibed using a harmonic or impulse kinematic excitation $w(t)$. The analysis included the influence of the control and disturbance on the behavior of the system. Both the control and disturbance forces have been assumed to act at the point connecting the car body and the suspension.

The oscillation of the system around the static equilibrium point can be given by a set of the two second-order differential equations

$$
\begin{aligned}
& m_{w} \ddot{y}_{w}=-c_{w}\left(\dot{y}_{w}-\dot{w}\right)-k_{w}\left(y_{w}-w\right)+c_{b}\left(\dot{y}_{b}-\dot{y}_{w}\right)+k_{b}\left(y_{b}-y_{w}\right)-u-z \\
& m_{b} \ddot{y}_{b}=-c_{b}\left(\dot{y}_{b}-\dot{y}_{w}\right)-k_{b}\left(y_{b}-y_{w}\right)+u+z
\end{aligned}
$$

where $y_{w}$ and $y_{b}$ are the displacement of the non-spring-supported and spring-supported mass, respectively. The parameters $k_{w}$ and $c_{w}$ define the stiffness and damping of the wheel, while $k_{b}$ and $c_{b}$ are the same for the passive vibro-isolation system.

By configuring the velocities and displacements of both masses to be included in the state vector $\mathbf{x}=\left[y_{w}, y_{b}, \dot{y}_{w}, \dot{y}_{b}\right]^{\mathrm{T}}$, Eqs. (2.1) can be written in the form of a first-order matrix differential equation

$$
\dot{\mathbf{x}}=\mathbf{A} \mathbf{x}+\mathbf{B}(u+z)+\mathbf{F} w
$$

where

$$
\begin{aligned}
\mathbf{A} & =\left[\begin{array}{cccc}
0 & 0 & 1 & 0 \\
0 & 0 & 0 & 1 \\
-\left(k_{w}+k_{b}\right) / m_{w} & k_{b} / m_{w} & -\left(c_{w}+c_{b}\right) / m_{w} & c_{b} / m_{w} \\
k_{b} / m_{b} & -k_{b} / m_{b} & c_{b} / m_{b} & -c_{b} / m_{b}
\end{array}\right] \\
\mathbf{B} & =\left[\begin{array}{c}
0 \\
0 \\
-1 / m_{w} \\
1 / m_{b}
\end{array}\right] \quad \mathbf{F}=\left[\begin{array}{c}
0 \\
0 \\
k_{w} / m_{w} \\
0
\end{array}\right]
\end{aligned}
$$

\section{SMC continuous regulation algorithm}

Figure 1 shows a block diagram of the system with the sliding mode control (Sam and Osman, 2005; Chen and Huang, 2005; Sam et al., 2008).

In order to increase the ride comfort, the parameters of the regulator are adjusted using the minimization condition for the variables that describe the movement of the spring-supported mass. The reduction of the car body vibration can be accomplished by introducing the following sliding plane

$$
S=\mathbf{D} \mathbf{x}
$$




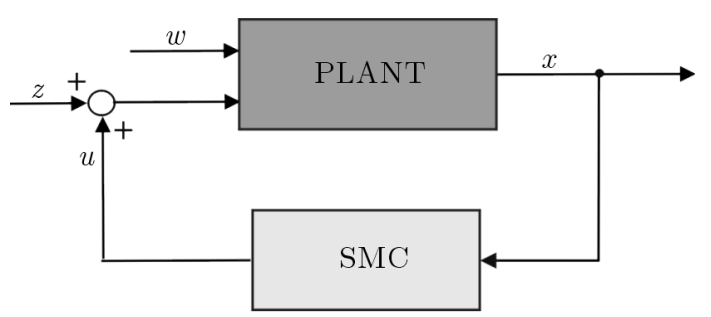

Fig. 1. Block diagram of a continuous SMC

where the transposed vector $\mathbf{D}$ is defined as follows

$$
\mathbf{D}=[0, \kappa, 0,1]
$$

This definition of the vector $\mathbf{D}$ should provide the minimal displacement $x_{2}=y_{b}$ and velocity $x_{4}=\dot{y}_{b}$ of the spring-supported mass. From the dynamical condition of the ideal sliding motion

$$
\dot{\mathbf{S}}=\mathbf{D} \dot{\mathbf{x}}=\mathbf{D}[\mathbf{A x}+\mathbf{B}(u+z)+\mathbf{F} w]=\mathbf{0}
$$

the equivalent (compensation) control $u^{e q}(t)$ is obtained, which after the omission of the excitation and disturbance influence can be written using the formula

$$
u^{e q}(t)=-(\mathbf{D B})^{-1} \mathbf{D A} \mathbf{x}
$$

An additional switching control $u^{s w}(t)$ is also included in order to guarantee the stability of the system. The control $u^{s w}$ is discontinuous with the sliding plane set as the switching line. This control can be calculated from the equation

$$
u^{s w}(t)=-K^{s w}(\mathbf{D B})^{-1} \operatorname{sgn}(\mathbf{D x})
$$

The sliding mode control is the sum of both of these components

$$
u(t)=u^{e q}(t)+u^{s w}(t)
$$

The sliding control realized in accordance to the algorithm presented above operates by dynamical compensation of the suspension stiffness and the introduction of additional damping. The switching component of control keeps the system close to the sliding plane. If the constant $K^{s w}$ is large enough, then the system becomes resistant to inaccuracies of the model. In practice, in order to limit the excessive switching ("chattering" effect) the signum function present in Eq. (3.5) is substituted by its approximation, e.g. "saturation" function. For the purpose of numerical calculations performed in this study, an arctangent approximation function has been used.

\section{SMC discrete regulation algorithm}

Due to the fact that the modern control systems are more often realized using digital devices, the sliding algorithm presented above needs to be modified. In order to choose the parameters for the discrete sliding mode controller, the following discrete equations are used (Yu et al., 2004; Yan and Fan, 2012)

$$
\mathbf{x}_{k+1}=\mathbf{A}_{d} \mathbf{x}_{k}+\mathbf{B}_{d}\left(u_{k}+z_{k}\right)+\mathbf{F}_{d} w_{k}
$$

where

$$
\mathbf{A}_{d}=\mathbf{\Phi}\left(T_{s}\right) \quad \mathbf{B}_{d}=\mathbf{\Psi B} \quad \mathbf{F}_{d}=\mathbf{\Psi} \mathbf{F}
$$


with $T_{s}$ being the sampling time. The matrix functions $\boldsymbol{\Phi}$ and $\boldsymbol{\Phi}$ are defined as

$$
\boldsymbol{\Phi}(\tau)=\exp (\mathbf{A} \tau) \quad \boldsymbol{\Psi}=\left[\mathbf{\Phi}\left(T_{s}\right)-\mathbf{I}\right] \mathbf{A}^{-1}
$$

The sliding plane can be chosen in the similar form as before (3.1)

$$
\mathbf{S}_{k}=\mathbf{D} \mathbf{x}_{k}
$$

By introducing

$$
f_{k}=\mathbf{B}_{d} z_{k}+\mathbf{F}_{d} w_{k}
$$

from the condition

$$
\mathbf{S}_{k+1}=\mathbf{D} \mathbf{x}_{k+1}=\mathbf{D}\left(\mathbf{A}_{d} \mathbf{x}_{k}+\mathbf{B}_{d} u_{k}+f_{k}\right)=0
$$

the equivalent control can be found

$$
u_{k}^{e q}=-\left(\mathbf{D B}_{d}\right)^{-1} \mathbf{D}\left(\mathbf{A}_{d} \mathbf{x}_{k}+f_{k}\right)
$$

Usually, $f_{k}$ component, whose value changes depending on the unknown excitation and disturbance, can be omitted, and the equivalent control component can be calculated from the equation

$$
u_{k}^{e q}=-\left(\mathbf{D B} \mathbf{B}_{d}\right)^{-1} \mathbf{D} \mathbf{A}_{d} \mathbf{x}_{k}
$$

In order to eliminate the component $f_{k}$ from Eq. (4.7), an assumption is made that its value varies only slightly from the value in the previous step, giving $f_{k} \approx f_{k-1}$. By using additionally discrete state equation (4.1), it can be shown that

$$
f_{k} \approx f_{k-1}=\mathbf{x}_{k}-\mathbf{A}_{d} \mathbf{x}_{k-1}-\mathbf{B}_{d} u_{k-1}
$$

After transformation, the final form of the equation describing the equivalent control can be written

$$
u_{k}^{e q}=u_{k-1}-\left(\mathbf{D B}_{d}\right)^{-1}\left[\left(\mathbf{D} \mathbf{A}_{d}+\mathbf{D}\right) \mathbf{x}_{k}-\mathbf{D} \mathbf{A}_{d} \mathbf{x}_{k-1}\right]
$$

The switching component usw is defined by a similar equation as Eq. (3.5)

$$
u_{k}^{s w}=-K^{s w}\left(\mathbf{D B}_{d}\right)^{-1} \operatorname{sgn}\left(\mathbf{D}_{d} \mathbf{x}_{k}\right)
$$

where the final control is the sum of both components.

(a)

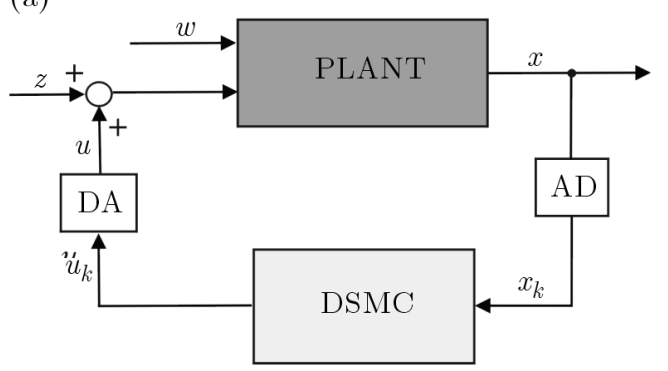

(b)

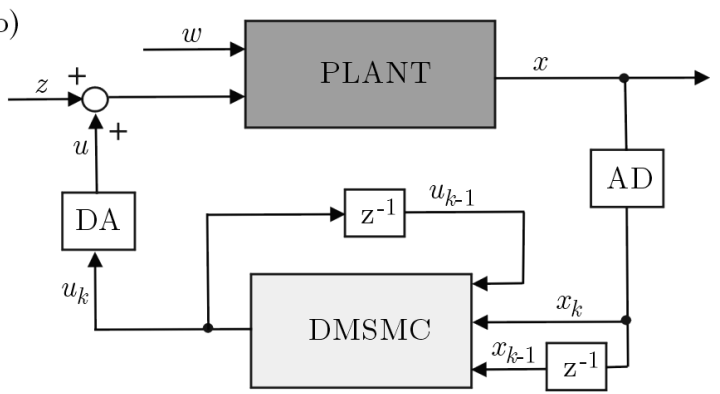

Fig. 2. Block diagrams of the discrete system $(\mathrm{AD}$ - analog/digital converter, DA - digital/analog converter): (a) DSMC, (b) DMSMC

Using numerical calculations, the efficiency of the DSMC regulator (Fig. 2a) with the equivalent control obtained using formula (4.8) is compared with the efficiency of the modified DMSMC regulator (Fig. 2b) related to equation (4.10). 


\section{Results of numerical calculations}

The emphasis of the numerical simulation is placed on finding the influence of the disturbance on the behavior and efficiency of the continuous SMC, discrete DSMC and DMSMC controllers. The following parameter values are chosen for matrices $(2.3): m_{w}=28 \mathrm{~kg}, m_{b}=510 \mathrm{~kg}$, $k_{w}=180000 \mathrm{~N} / \mathrm{m}, k_{b}=20000 \mathrm{~N} / \mathrm{m}, c_{b}=1000 \mathrm{Ns} / \mathrm{m}$. The analysis is limited to the response of the system under the harmonic excitation of amplitude $a_{0}=0.005 \mathrm{~m}$ and angular frequency $\omega=8.396 \mathrm{rad} / \mathrm{s}$ (first vibration mode of the passive system without control) and to the impulse excitation described in detail later on.

Several dimensionless parameters are introduced: $\beta_{x}, \beta_{v}, \gamma, \tau_{s}$ and defined using equations: $\beta_{x}=\kappa_{x} K_{x}, \beta_{v}=\kappa_{v} K_{v}, \gamma=K^{s w} / \omega_{0}$ and $\tau_{s}=T_{s} / T_{0}$, where: $K_{x}=a_{0}, K_{v}=\omega_{0} a_{0}, T_{0}=2 \pi / \omega_{0}$, and $\omega_{0}=\sqrt{k_{b} / m_{b}}$ correspond to the first vibration mode of the system. The function $\operatorname{sgn}(S)$ present in Eqs. (3.5) and (4.11) is substituted by continuous $2 / \pi \arctan (\eta S)$ with $\eta=100$ used for the calculations.

It is assumed that the disturbance is in the form of a Gaussian white noise of zero average value and variance of value $25 \mathrm{~N}^{2}$ (standard deviation $s_{0}=5 \mathrm{~N}$ ). An exemplary realization of this disturbance for the sampling time $T_{s}=0.05 T_{0}$ is shown in Fig. 3 .

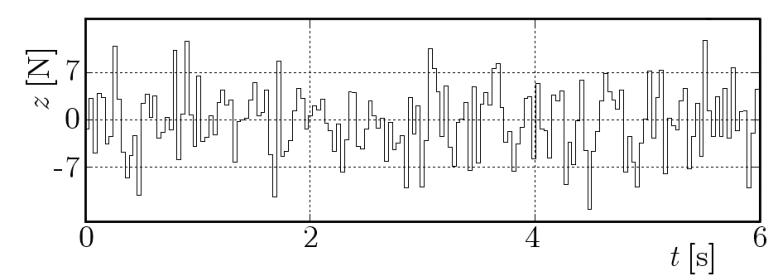

Fig. 3. An example of the disturbance signal

Figure 4 shows the displacement of the spring-supported mass for the continuous SMC, discrete DSMC, Eq. (4.8), and modified discrete DMSMC regulator, Eq. (4.10). For the discrete control, the sampling time was $T_{s}=0.02 T_{0}\left(\tau_{s}=0.02\right)$. By comparing the results, one can notice that in the case of discrete control, the response looks periodic in contrast to the aperiodic continuous control SMC. On the assumed control parameters, the efficiency of SMC and DMSMC regulator is comparable.

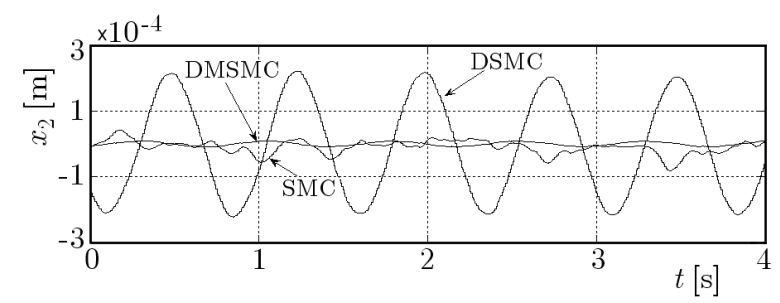

Fig. 4. Displacement plot: SMC $\left(\beta_{x}=\beta_{v}=1, \gamma=0.1\right)$, DSMC $\left(\beta_{x}=\beta_{v}=1, \gamma=0.001\right)$, $\operatorname{DMSMC}\left(\beta_{x}=\beta_{v}=1, \gamma=0.001\right)$

The irregularity seen in the response to the SMC regulator is caused by the high sensitivity of the system to the external disturbance. This fact is supported by Fig. 5 in which the response to three disturbance signals of different standard deviations $s=5,10$ and $15 \mathrm{~N}$ is shown (for $s=n s_{0}, n=1,2,3, s_{0}=5 \mathrm{~N}$ ). The change in the response to these disturbances is nearly linear (with linear rise of the disturbance). Although the amplitude of vibration can be attenuated by increasing the value of parameters $\beta_{x}$ and $\gamma$, setting them too high is undesirable due to the effect of "chattering", and also due to the time delay present between the regulator and the actuator.

In the case of the modified DMSMC digital controller, the response to the disturbance is definitely lower. When using the same values of $n$ that define the disturbance as before, 


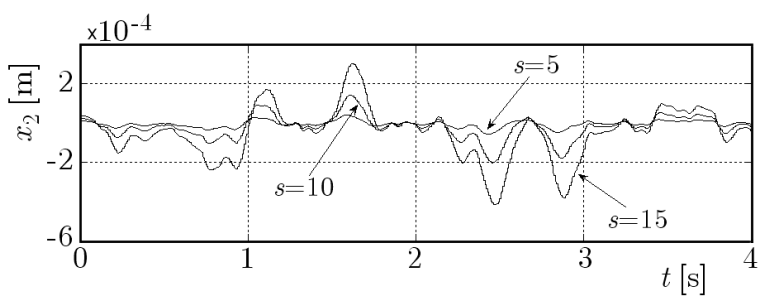

Fig. 5. Influence of the disturbance on the displacements $-\operatorname{SMC}\left(\beta_{x}=\beta_{v}=1, \gamma=0.1\right)$

the difference between the responses is practically undetectable. Therefore, Fig. 6 shows the displacement for $n=1$ and $n=20$ (with $\tau_{s}=0.02$ ). Even with twenty times higher value of $s$ $(s=5 \mathrm{~N}$ and $100 \mathrm{~N})$, the differences are still minor, which means that the DMSMC system is resistant to the disturbance.

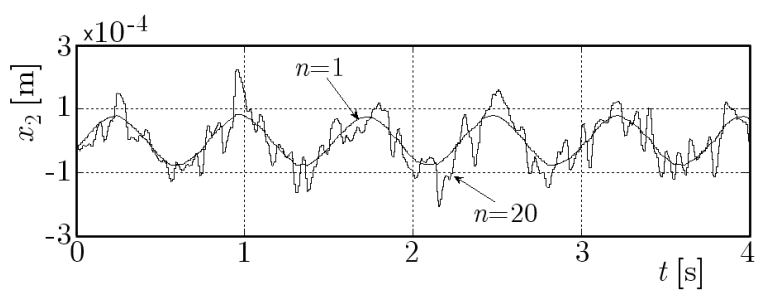

Fig. 6. Influence of the disturbance on the displacements $-\operatorname{DMSMC}\left(\beta_{x}=\beta_{v}=1, \gamma=0.001\right)$

The efficiency of the discrete regulator is decreased when the sampling time increases (Fig. 7). The presented displacements are obtained for the DMSMC with different sampling times $\tau_{s}=0.01,0.02,0.03$ and $\beta_{x}=\beta_{v}=1, \gamma=0.001$ and $s=5 \mathrm{~N}$. The relation of the amplitude of vibration with the sampling time is nonlinear, e.g. a change in $\tau_{s}$ from 0.01 to 0.02 leads to the amplitude rise by ten times. When rise of $\tau_{s}$ is continued, the amplitude of vibration is changed by a smaller factor. On the other hand, too small sampling time is undesirable due to computation time taken for the realization of digital control.

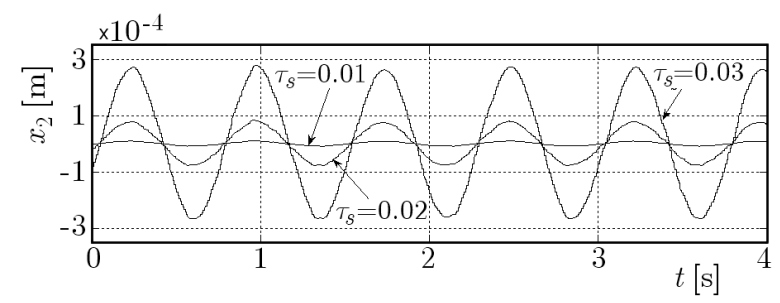

Fig. 7. Relation between the sampling time and displacements - DMSMC $\left(\beta_{x}=\beta_{v}=1, \gamma=0.001\right)$

Figure 8 shows the displacements (Fig. $8 \mathrm{a}-$ for $\beta_{x}=0.1,1,2$ ) and velocities (Fig. $8 \mathrm{~b}-$ for $\beta_{x}=1$ only $)$ of the spring-supported mass in the DMSMC system $\left(\beta_{v}=1, \gamma=0.001, \tau_{s}=0.02\right)$.

With the rise of value $\beta_{x}$, the maximal displacements are decreased but the maximal velocities and accelerations are increased. In addition to that, the character of vibration is changed, with more influence of high-frequency component present in the system. For high enough values of $\beta_{x}$, the chattering effect starts to occur.

In order to test the behavior of the vibroisolation system, when crossing an obstacle, analysis of the response to the impulse excitation has been performed.

To describe the excitation in simulations, the bump "rounded pulse" function has been used. It is defined as follows (Shekhar et al., 1999)

$$
w(t)=\frac{1}{4} h\left[e \eta\left(t-t_{0}\right)\right]^{2} \exp \left[-\eta\left(t-t_{0}\right)\right] H\left(t-t_{0}\right)
$$



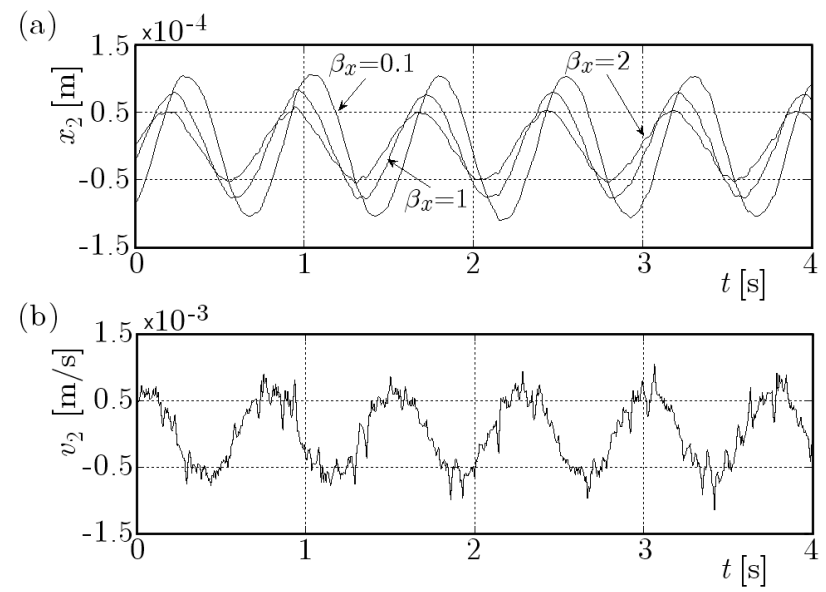

Fig. 8. Effect of the parameter $\beta_{x}$ - DMSMC system $\left(\beta_{v}=1, \gamma=0.001, \tau_{s}=0.02\right)$ : (a) displacements, (b) velocities $\left(\beta_{x}=1\right)$

where $H\left(t-t_{0}\right)$ is the Heaviside function. Equation (5.1) has continuous first and second derivates and the maximal value equal to $h$ (Fig. 9). The parameter $\eta$ defines the sharpness of the impulse. At $\eta=\omega_{n 1}=8.396 \mathrm{rad} / \mathrm{s}$, the impulse duration is equal to the half of the first vibration mode period. In numerical calculations, the value $h$ has been chosen to be equal to $0.05 \mathrm{~m}$.

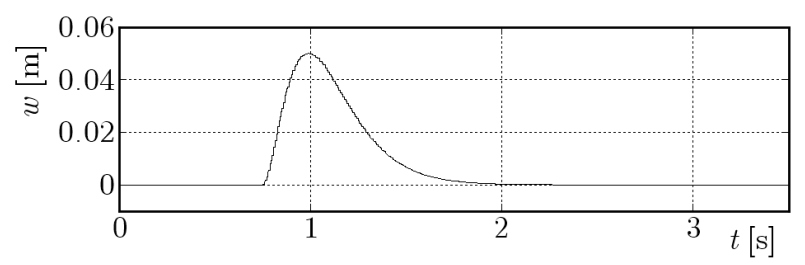

Fig. 9. Impulse excitation $(\gamma=8.396 \mathrm{rad} / \mathrm{s}, h=0.05 \mathrm{~m})$

Figure 10 shows the response of the SMC $\left(\beta_{x}=\beta_{v}=1, \gamma=0.1\right)$ and DMSMC $\left(\beta_{x}=\beta_{v}=1\right.$, $\gamma=0.001$ ) system to impulse excitation (5.1). In both cases, the rate of displacement reduction is similar. In the case of the SMC, the damping of the disturbance (Fig. 10a) is slower when considered in relation to DMSMC, but it is also less steep, see the velocity plot in Fig. 10b.
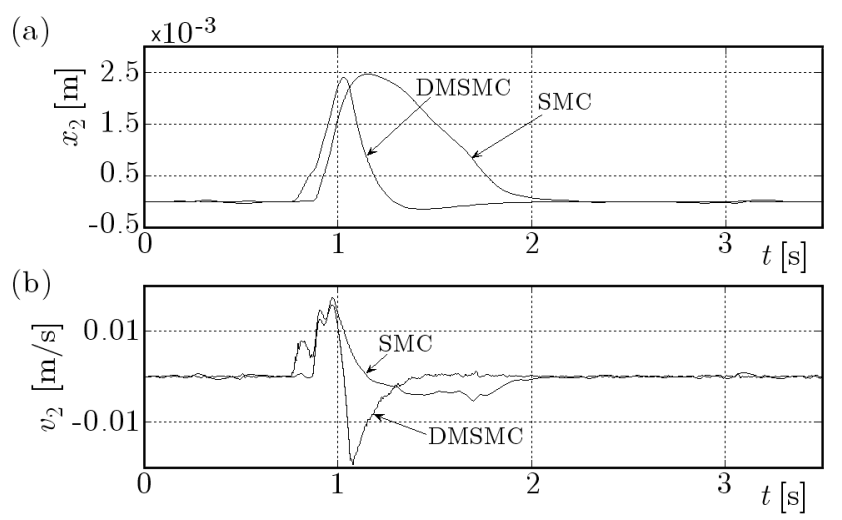

Fig. 10. Response to the impulse excitation - regulators: SMC $\left(\beta_{x}=\beta_{v}=1, \gamma=0.1\right)$ and DMSMC $\left(\beta_{x}=\beta_{v}=1, \gamma=0.001\right):(\mathrm{a})$ displacements, (b) velocities

In the considered example, if the value of $\gamma$ is too high (for $\gamma>0.01, \beta_{x}=1$ ), the "chattering" effect occurs (Fig. 11). Apart from the additional high-frequency oscillations, the changes in the value are twice as high as for the system with optimally chosen parameters of control (Fig. 10a). 
In addition to that, an increase in the value of $\beta_{x}$, causes the possibility of occurring of the chatter to be higher (at $\beta_{x}>10$, the chattering can be observed for $\gamma=0.001$ ).

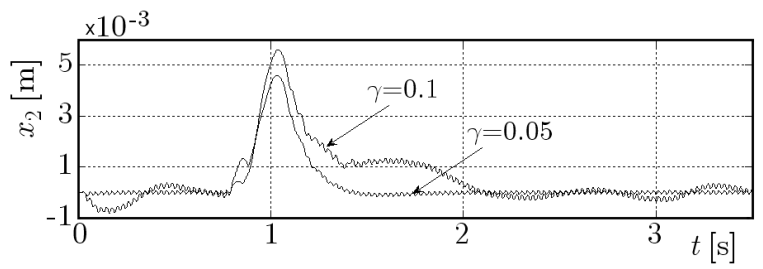

Fig. 11. Displacement plot with chattering $\left(\beta_{x}=\beta_{v}=1, \gamma=0.05,0.1, \tau_{s}=0.02\right)$

\section{Summary}

The analysis of the results allows formulating of the following conclusions:

- In all the considered active systems, the reduction of vibration is well enough, at least within the range that includes the first vibration mode. As this mode is significant, when considering vibration of the car body, it should be noted that making use of the presented regulators greatly increases the driving comfort.

- The continuous SMC regulator, for very high values of parameters $\beta_{x}$ and $\gamma$, is theoretically more effective than discrete regulators. However, it is more susceptible to disturbances as well as the time delay present during control.

- From the two presented discrete regulators, the better one is definitely DMSMC. It is much more resistant to disturbances, and for small sampling times, its performance is comparable to SMC.

- When adjusting the parameters of sliding mode regulators, the possibility of the "chattering" effect to occur should be taken into consideration. Values of the regulator parameters should allow the optimal and regular operation of the regulator without undesirable highfrequency oscillations.

\section{References}

1. Chen P.-C., Huang A.-C., 2005, Adaptive sliding control of non-autonomous active suspension systems with time-varying loadings, Journal of Sound and Vibration, 282, 1119-1135

2. HuAng S.-J., Chen H.-Y., 2006, Adaptive sliding controller with self-tuning fuzzy compensation for vehicle suspension control, Mechatronics, 16, 607-622

3. Lee H., UtKin V.I., 2007, Chattering suppression methods in sliding mode control systems, Annual Reviews in Control, 31, 179-188

4. Lin J., Lian R.-J., HuAng C.-N., SiE W.-T., 2009, Enhanced fuzzy sliding mode controller for active suspension systems, Mechatronics, 19, 1178-1190

5. Łuczko J., Ferdek U., 2012, Comparison of different control strategies in a semi-active vehicle suspension system (in Polish), Czasopismo Techniczne, 11, 6-M, 81-92

6. Rajeswari K., Lakshmi P., 2008, GA tuned distance based fuzzy sliding mode controller for vehicle suspension systems, International Journal of Engineering and Technology, 5, 1, 36-47

7. Sam Y.M., Osman J.H.S., 2005, Modeling and control of the active suspension system using proportional integral sliding mode approach, Asian Journal of Control, 7, 2, 91-98

8. Sam Y.M., Osman J.H.S., Ghani M.R.A., 2004, A class of proportional-integral sliding mode control with application to active suspension system, Systems and Control Letters, 51, 217-223 
9. Sam Y.M., Suaib N.M., Osman J.H.S., 2008, Hydraulically actuated active suspension system with proportional integral sliding mode control, WSEAS Transactions on Systems and Control, $\mathbf{9}$, 3, 859-868

10. Shekhar N.C., Hatwal H., Mallik A.K., 1999, Performance of non-linear isolators and absorbers to shock excitations, Journal of Sound and Vibration, 227, 2, 293-307

11. Snamina J., Kowal J., Wzorek T., 2011, Analysis of energy dissipation in vehicle suspensions for selected control algorithms (in Polish), Czasopismo Techniczne, 2, 1-M, 233-240

12. YAN M., FAN L., 2012, Discrete sliding mode control for DC-DC converters with uncertainties, Przeglad Elektrotechniczny, 88, 5b, 60-63

13. Yoshimura T., Kume A., Kurimoto M., Hino J., 2001, Construction of an active suspension system of a quarter car model using the concept of sliding mode control, Journal of Sound and Vibration, 239, 2, 187-199

14. Yu W.-C., Wang G.-J., Chang C.-C., 2004, Discrete sliding mode control with forgetting dynamic sliding surface, Mechatronics, 14, 737-755

Manuscript received December 12, 2014; accepted for print March 31, 2015 